\title{
ECONOMIC ANALYSIS OF TECHNOLOGY AND PROPERTIES OF LEGENDRE-FENCHEL TRANSFORMATIONS
}

\author{
ILKO VRANKIĆ, JASMINKA ŠOHINGER, MIRA KRPAN
}

\begin{abstract}
:
This paper examines a two-way relationship between convex analysis and microeconomic theory. Motivation for this paper are the observed similarities in the structure of the theory of consumer behavior and production theory. The fact that the behavior of variables is not determined by their nature but, rather, by their relationships is best illustrated and explained by using convex sets and convex analysis, which occupy central place in microeconomic theory. This paper is the result of efforts to make complex results of convex analysis and its application in microeconomic theory more transparent.

Starting with the well-known economic phenomenon of profit maximization the authors derive in a novel way general results within the framework of convex analysis. From those results follow, directly and indirectly, the conclusions of the theory of consumer and producer behavior. The authors show that applying the Fundamental Theorems of Calculus opens up a new perspective in which the marginal cost curve can be interpreted as the marginal profit curve. This enables the derivation of Hotelling's lemma in a new way. Using the new interpretation of Hotelling's lemma, the authors reconstruct the cost function and confirm the Conjugate Duality Theorem of Legendre-Fenchel transformations. Relaxing the assumption of differentiability by describing the graph of the cost function as the envelope of its tangents, the authors rederive the properties of Legendre-Fenchel transformations and show that they hold in general. The path from the well-known economic facts to completely general conclusions of convex analysis is continued by applying the Conjugate Duality Theorem of Legendre-Fenchel transformations to the profit function. The essence of the dual characterization of technology by the profit function is illustrated by the graphical representation of linear homogeneity of the profit function. It results in the possibility to reconstruct the production function while using only the First Order Conditions to rederive Hotelling's lemma. It is this inductive-deductive approach used to examine the properties of Legendre-Fenchel trasformations and their application in the theory of consumer and producer behavior that establishes a two-way relationship between convex analysis and microeconomic theory.
\end{abstract}

\section{Keywords:}

Hotelling's lemma, Legendre-Fenchel transformations, convexity, duality, inversion, sub-differentiability, monotonicity, cyclical monotonicity.

JEL Classification: C02, D01, D21

\section{Authors:}

ILKO VRANKIĆ, Faculty of Economics and Business Zagreb, Croatia, Email: ivrankic@efzg.hr JASMINKA ŠOHINGER, Faculty of Economics and Business Zagreb, Croatia, Email: jsohinger@efzg.hr MIRA KRPAN, Faculty of Economics and Business Zagreb, Croatia, Email: moraic@net.efzg.hr 


\section{Citation:}

ILKO VRANKIĆ, JASMINKA ŠOHINGER, MIRA KRPAN (2019). Economic Analysis of Technology and Properties of Legendre-Fenchel Transformations. International Journal of Economic Sciences, Vol. VIII(2), pp. 159-183., 10.20472/ES.2019.8.2.011 


\section{Introduction}

Consumer theory and theory of production play a pivotal role in microeconomic theory. In this context particularly important is the dual approach, where the same economic phenomenon can be described by different sets of variables. It may appear that this would make the analysis more complicated because of a big number of relationships that the dual approach produces. However, given the fact that numerous microeconomic problems have the same structure, it can also simultaneously enrich and simplify the analysis as well as have the didactical and methodical significance. Relationships between various primal and dual functions that describe preferences and technology and important duality results can be found in Blackorby et al. (1978), while Weymark (1980) analyses duality relationships in consumer theory.

Duality theory in production enables the firm's technology to be described equally well with the production function as well as with the corresponding value functions (cost, revenue and profit functions) under certain regularity conditions (Diewert, 1982). As two main practical advantages of duality theory in economics, Diewert emphasizes deriving systems of demand equations which are consistent with maximizing or minimizing behavior by differentiating a function, as opposed to solving explicitly a constrained maximization or minimization problem and deriving "in an effortless way the comparative statics theorem originally deduced from maximizing behavior" (Diewert, 1982). In his comments to Diewert's survey of the applications of duality theory in economics, Lawrence J. Lau emphasizes that, although there are many alternative approaches to duality theory, they can be classified into three groups. The first approach is "based on the conjugacy correspondence developed by Fenchel $(1949,1953)$ and extended by Rockafellar (1970a)", (Diewert, 1974). The second group of approaches "is based on the symmetric duality between gauge functions, or distance functions, or polar cones of convex sets", (Diewert, 1974), whose pioneer was Shepard (1953), and the third group of approaches "is based on the duality between the set of production possibilities and its support functions", (Diewert, 1974), whose pioneers were Uzawa (1964), McFadden (1966) and Diewert (1971, 1973).

Duality theory has been widely applied empirically in the estimation of the technology parameters without the need to explicitly specify the technology but to start with the functional form of the value function for which the researcher has data, or where the estimation is easier. This empirical application of duality theory was especially intensified after the development of flexible functional forms for the dual cost and profit functions (Diewert, 1971, Christensen, Jorgenson and Lau, 1971, Sargan, 1971). In his evaluation of contributions to dual modelling in production from 1982 Shumway (1995) mentions more than 100 studies that "use neoclassical duality concepts to specify an economic model of production". Appelbaum (1978) and Burgess (1975) were among the first that tested duality theory in empirical applications and found that "primal and dual do not yield 
similar implications" (Appelbaum, 1978). Lusk et al. (2002) analyze the effect of a particular dataset on dual relationship between the restricted profit, unrestricted profit and production function using Monte Carlo technique and showed that Lau's Hessian identities failed to hold in the presence of measurement error and low relative price variability. In their econometric estimation with the generated pseudo-dataset Rosas and Lence (2015) analyse the ability of the dual approach to recover the technology features and show that the dual approach is not able to provide "parameter estimates that are sufficiently close to their true values", (Rosas \& Lence, 2015).

Unlike testing duality theory in empirical context, analysis in this paper brings back the attention to the theory and the origins of duality in microeconomic theory. The main characteristic of the dual approach is the switching of the variables (Roy, 1942). A natural connection between quantities and prices that was recognized already by Hotelling (1932), who first mentioned the notion of duality in economic literature, is according to Blume (2008a, 2008b) deeply rooted in convex analysis and in different descriptions of convex sets. For economists convex analysis is full of applications and at the same time analytically complex. This difficulty can be overcome by the inductive-deductive approach examining the properties of Legendre-Fenchel transformations. The mathematical treatment of convex analysis and Legendre-Fenchel transformations can be found in Rockafellar (1970). Lau (1978) in his analysis of duality between the production function and the normalized profit function defines Legendre transformations as "a change of variables of a function from point coordinates to plane coordinates."

The fundamental question of this paper is whether the familiar equality of price and marginal cost in the microeconomic analysis of a perfectly competitive firm can serve as a starting point for the inductively derived general results of convex analysis from which, indirectly and directly, follow the results of the theory of consumer behavior and the theory of production. The starting point in this paper is a convex cost function. By applying the Fundamental Theorems of Calculus the marginal cost curve can be interpreted from a new perspective - as the marginal profit curve. This opens a new way in which Hotelling's lemma can be obtained. According to Hotelling's lemma the derivative of the profit function with respect to the price of good $i$ gives the competitive firm's net supply function of that good. (Mas-Colell et al., 1995). In the standard advanced microeconomics texts Hotelling's lemma is proved via the envelope theorem. In the convex analysis framework Blume in his review of duality in microeconomics describes the convex version of Hotelling's lemma as a consequence of the inversion property of subdifferentials for concave and convex functions (Blume, 2008a, 2008 b). In our paper, heuristically, Hotelling's lemma is interpreted as the First Order Condition in an optimization problem that enables the reconstruction of a cost function from a profit function. Through novel interpretations of the marginal profit and the corresponding geometry we derive the dual of the profit maximization model, which confirms the Conjugate Duality Theorem of Legendre-Fenchel transformations. 
Two descriptions of equilibria of a profit maximizing firm are connected by the inversion property of the Legendre-Fenchel transformations. In our analysis the relationship between the solutions of the dual optimization problems does not depend on the differentiability of the dual functions. Instead, the assumptions include convexity and closedness of the epigraph of the cost and profit functions. By describing the graph of a convex function as an envelope of its tangents, conclusions are made in the economic context that confirm the convexity of Legendre-Fenchel transformations, the Conjugate Duality Theorem of Legendre-Fenchel transformations, their inversion and differentiability properties as well as their properties of monotonicity and cyclical monotonicity. Those properties are important in their application to numerous microeconomic phenomena. In particular, there is the application of the Conjugate Duality Theorem to a convex indicator of the closed and convex set of technologically available production plans, which enables us to see the essence of duality in the dual description of technology by the profit function. The strength of the dual description of the set of technologically available production plans is reflected in the reconstruction of the production function, the process in which Hotelling's lemma appears again as the necessary First Order Condition.

The new approach has another advantage. By representing technology with a convex indicator function of the set of technologically feasible production plans a new dimension opens up. We can now graphically represent the property of linear homogeneity of the profit function by intercept values on the profit axis of the supporting hyperplanes determined by the proportional prices.

It is said that science is organized knowledge. In this paper microeconomic theory, calculus, and convex analysis are connected in a unique way. In his work Blume (2008a, 2008b) takes up general results on the properties of Legendre-Fenchel transforms and applies them deductively in microeconomic theory. Difficulties in understanding general results of convex analysis represent the motivation for the inductive approach in this paper, which begins with the well-known economic problem of profit maximization. Applying the Fundamental Theorems of Calculus authors derive Hotelling's lemma in the simplest way not previously contained in economic theory. Hotelling's lemma is key for the changing places of variables, which enables the reconstruction of the minimum cost function in a novel way. Based on the dual description of convex sets and using graphical representations in the economic context authors derive completely general results of convex analysis. These results are then inductively used to directly and indirectly derive all results of the theory of consumer and producer behavior. In this way the deductive approach in literature is enriched by the inductive approach that precedes it.

The analysis itself is graphical and it contains economic interpretations that at first glance appear unusual. However, it reveals clear relationships among functions describing technology on perfectly competitive markets. In addition to the theoretical contributions towards understanding ways in which different sets of variables describe microeconomic 
phenomena under perfect competition, this approach could equivalently be used in the analysis of other market structures. The new approach is actually a new method.

The remainder of the paper is organized as follows. The next section starts with the profit maximization model in the perfect competition framework. It analyzes the marginal cost curve from the new perspective giving it another interpretation as the marginal profit curve. In it Hotelling's lemma is derived in a new way. The new interpretation of Hotelling's lemma is used as a starting point in reconstructing the cost function and confirming the Conjugate Duality Theorem of Legendre-Fenchel transformations. The following third section is devoted to the derivation of the properties of Legendre-Fenchel transformations by enveloping the cost curve with its tangents and without using the differentiability assumption. The fourth section applies the Conjugate Duality Theorem to the indicator function of the set of feasible production plans revealing the essence of duality in the dual description of technology by the profit function. It finishes with the graphical representation of linear homogeneity of the profit function. The final section summarizes the obtained findings.

\section{Heuristic considerations}

The analysis in this chapter is based on two Fundamental Theorems of Calculus. Those two theorems connect the problems of the tangent and that of the area. The problem of the tangent is solved by the derivative explaining the slope. The problem of the area is solved by the definite integral. Varying the upper limit of the integral yields the function of the area. According to the First Fundamental Theorem of Calculus the derivative of the function of the area shows the graph that bounds that area. The Second Fundamental Theorem of Calculus expresses the area bounded by the graph of the derivative of some function within given boundaries by changing the values of the function. This result is also called the Newton-Leibnitz formula.

For completeness of the analysis in the first half of this chapter we represent profit of a competitive firm by an area based on the Newton-Leibnitz formula. This approach enables clear demonstration of how the profit of the firm rises as quantity rises until the First Order Condition according to which price equals marginal cost is fulfilled. Different equilibria of the firm reached at different prices define maximum profit of the firm as a function of the area. This is how applying the First Fundamental Theorem of Calculus shows how Hotelling's lemma can be derived in a new way. This approach has another advantage: it makes it possible to reconstruct the cost function by simply changing places of the variables, which at the same time confirms the Theorem of Duality of the LegendreFenchel transformations.

The competitive firm's profit maximization model is represented by 


$$
\pi(p)=\max _{y \geq 0} p y-c(y)
$$

where $p$ is the product price, $y$ is the quantity of production, $c(y)$ is the cost function and $\pi(p)$ is the profit function. In the first part of the analysis we assume the cost function to be convex. The perfectly competitive firm's long run supply function, $y(p)$, is derived from the usual First Order (necessary) Condition according to which price equals marginal cost in equilibrium, while the marginal cost function is increasing,

$$
p=\frac{\partial c}{\partial y}
$$

(2)

This result can also be obtained graphically by applying the Newton-Leibniz equation which is illustrated in Figure 1.

Figure 1: Hotelling's lemma

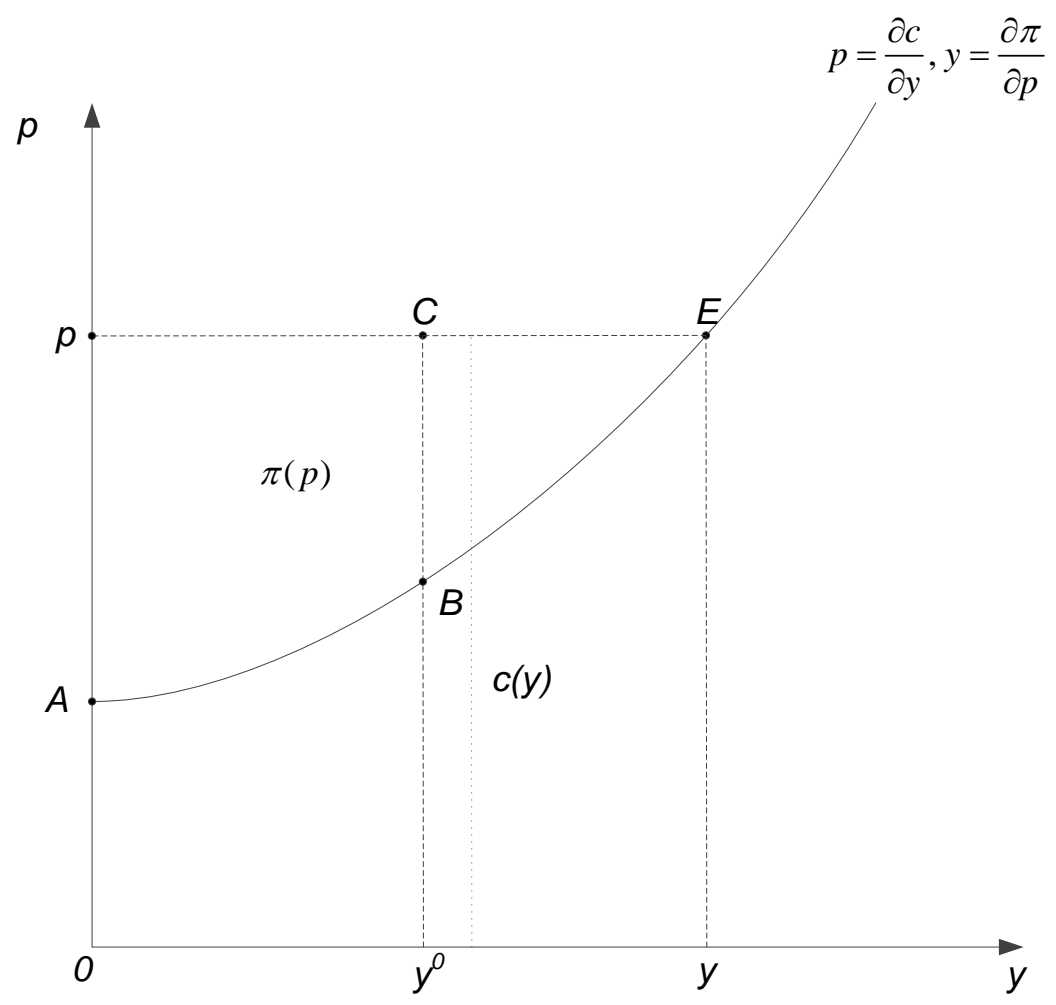

Source: Authors 
For the given product price, $p$, higher than the minimum marginal cost, let's observe the quantity of production $y^{0}$, for which marginal costs are lower than the price. Total revenue is represented by the area $0 y^{0} \mathrm{Cp}$. According to the Newton-Leibniz equation applied to this case, the area under the marginal cost curve is equal to the change in total costs. In the long run total costs are represented by the area $0 y^{0} B A$. Consequently, total profit is represented by the area $A B C p .^{1}$

If the quantity of production is increased by a small unit, the product price represents an increase in revenue or the additional revenue. This additional revenue is actually the additional gain represented by the height $y^{0} \mathrm{C}$. The above mentioned increase in production by a small unit also increases costs. The additional cost or the additional loss is represented by the height $y^{0} B$. Since the additional gain is higher than the additional loss, the firm keeps increasing production until the price is equal to the marginal cost. Total gain or total revenue is represented by the area $0 y E p$, total loss or total cost by the area $0 y E A$ and maximum profit by the area $A E p$.

Graphically, as we increase production from $y^{0}$ on while the price remains given, point B moves to the right along the marginal cost curve. At the same time point $C$ is also moving to the right but it is constantly above the point $\mathrm{B}$. Consequently, profit represented by the area $A B C p$ is increasing until points $B$ and $C$ become equal in the point $E$. Therefore, optimum production is that for which price equals marginal cost. If we continued to increase production beyond that point, B would surpass $C$ and it would be higher than $\mathrm{C}$. In this situation profit would be lower than maximum and the difference would be represented by the area ECB.

For every price higher than the point $A$ in Figure 1, the area to the left of the marginal cost curve or beneath it from the vertical axis perspective represents maximum profit. This change in perspective can be visually represented in the three dimensional space by rotating the plane of the marginal cost curve until it becomes horizontal which is represented in Figure 2.

\footnotetext{
${ }^{1}$ In the short run the analysis is devoted to short-run profit as the difference between revenue and variable costs.
} 
Figure 2: Marginal profit obtained by rotating marginal costs

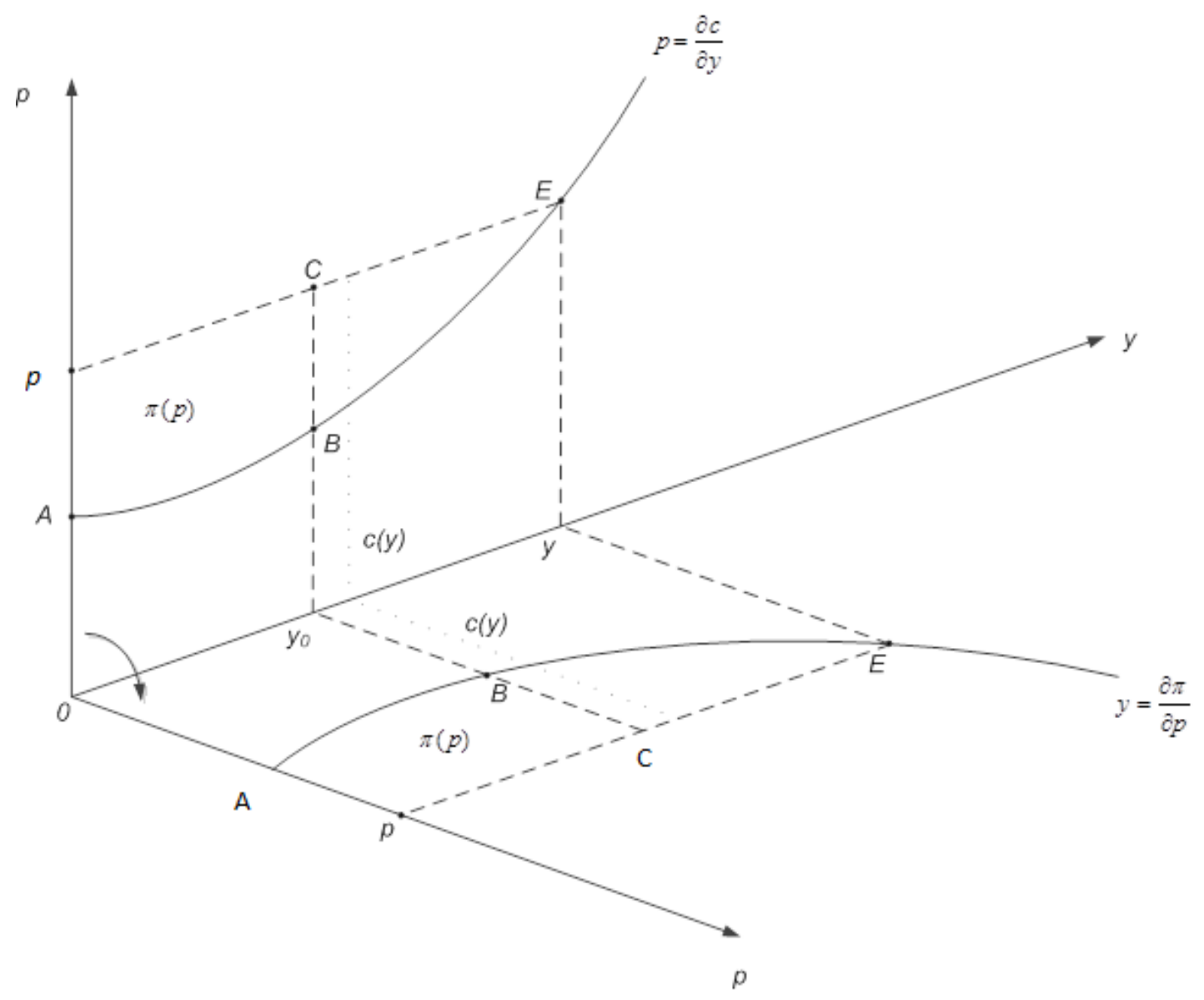

Source: Authors

As shown in Figure 2 the maximum profit function is clearly identified as the function of the area ApE above the marginal cost curve. By rotating and mirror-imaging the graph in Figure 2 we show that the marginal cost curve can be mapped into what can simultaneously be interpreted as the marginal profit curve. According to the First Fundamental Theorem of Calculus the mapped curve graphs the first derivative of the maximum profit function with respect to price. At the same time that curve describes the optimum production at the given price. This represents a new way in which optimum production can be obtained by deriving the maximum profit function with respect to price, or

$$
y=\frac{\partial \pi}{\partial p} .
$$


This result is known as Hotelling's lemma. It follows that the functions of marginal cost and marginal profit are inverse functions.

Figure 2 shows that by rotating the marginal cost curve onto the marginal profit curve variables change places. This clearly illustrates the essence of duality. When price and quantity change places, profit function and cost function do as well. This is how from model (1) we directly obtain

$$
c(y)=\max _{p \geq 0} p y-\pi(p) .
$$

It would be natural to ask if we could interpret Hotelling's lemma as the necessary First Order Condition of the previous optimization problem and how to justify it. To find answers to those questions we need a change in perspective. Before we were looking for the profit maximizing production level for the given price. Let us now try to determine the price at which the firm supplies the given level of quantity when only the profit function is known.

We take the quantity of production, $y$, as given and look at the price $p^{0}$ for which the marginal profit is lower than the quantity which is represented in Figure 3.

\section{Figure 3: The dual problem}

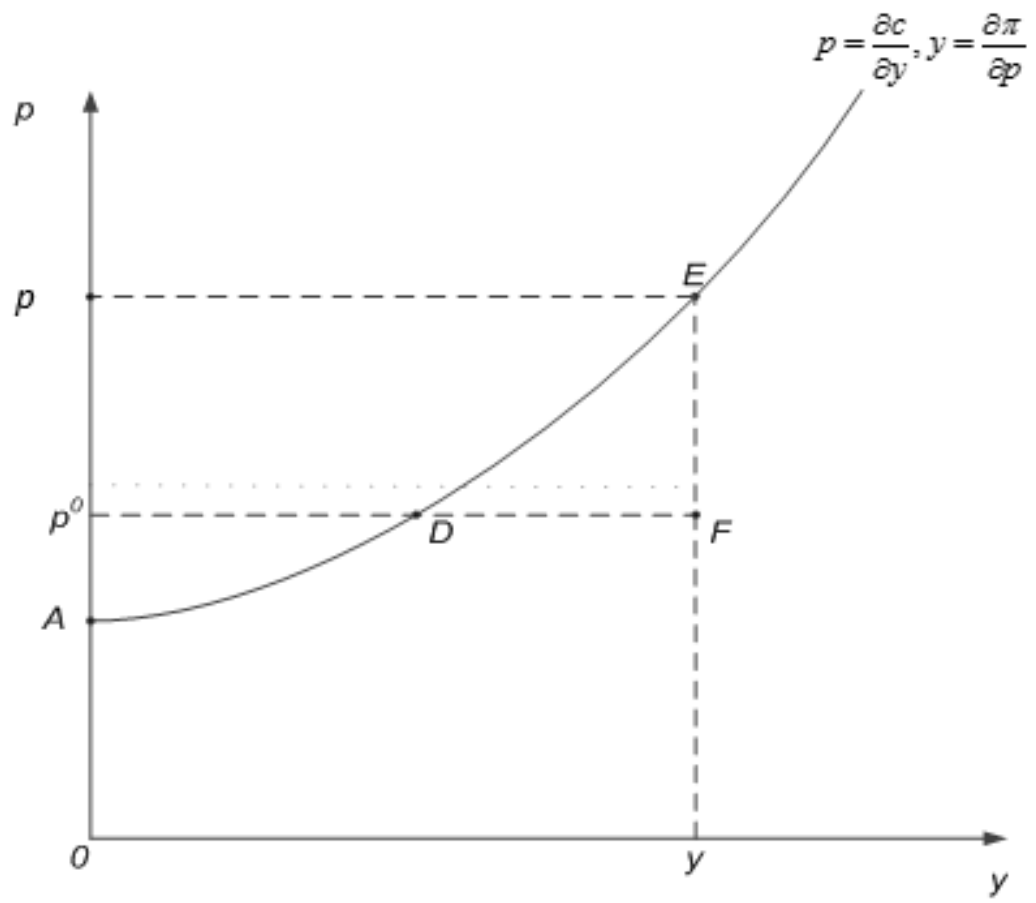

Source: Authors 
Now the decision variable is the price and the goal is to derive maximum profit for the given level of production. Total revenue is represented by the area $0 y F^{0}{ }^{0}$, and maximum profit by the area $A D p^{0}$. For maximum profit to be realized, costs should be represented by the area $0 y F D A$. Minimum costs are represented by the area $0 y E A$ and they cannot be decreased any further. This is why the product price will be increased by a small unit. Maximum profit is increased by $\frac{\partial \pi\left(p^{0}\right)}{\partial p}$ and now our goal is farther for the amount represented by the height $p^{0} D$. The greater distance from the goal represented by the marginal profit can be interpreted as the additional loss. The additional gain is an increase in revenue equal to the amount determined by the given production level or the height $p^{0} F$. It decreases the distance from our goal, which is to obtain the maximum profit for the given production level.

When the additional gain is higher than the additional loss, we keep increasing the price. In equilibrium represented by the point $E$ the additional loss or marginal profit is equal to the additional gain or the given level of production. So we find the price for which the difference between total gain and total loss or the difference between the revenue and the maximum profit is maximized. This difference represents minimum economic costs (4).

At first glance it may seem strange that the increase in maximum profit per small increase in price, or, marginal profit, is interpreted as loss. Graphical illustration of this way of thinking can help illustrate which important results can be derived this way.

By increasing the price from $p^{0}$ at the given quantity of production, point $\mathrm{D}$ moves along the marginal profit curve to the right (Figure 3). At the same time point $F$ moves upwards to remain to the right of the point $D$. Minimum costs of the given production level is represented by the area $0 y E A$. This area is the maximum of the difference between $0 y F p^{0}$ and the area $A D p^{0}$. Therefore, it is the difference between revenue and maximum profit at the price $p^{0}$. Minimum costs are achieved at the price for which points $\mathrm{D}$ and $\mathrm{F}$ meet at the point $\mathrm{E}$. This is the price which is equal to the marginal costs of production, or, where $p=\frac{\partial c}{\partial y}$. At the same time, at this price marginal profit is equal to the given level of production, $y=\frac{\partial \pi}{\partial p}$.

In this way the Hotelling's lemma is interpreted as the necessary First Order Condition for the optimization problem (4) that enables the reconstruction of the cost function.

In mathematical literature the transformations, 


$$
\pi(p)=\sup _{y} p y-c(y)
$$

(5a)

$$
c(y)=\sup _{p} p y-\pi(p) .
$$

are known as Legendre-Fenchel transformations ${ }^{2}$. By reconstructing the cost function we showed in a new way that by applying the Legendre-Fenchel transformation twice we get the original function, the one we started out from. In this way we confirmed the duality of Fenchel-Legendre tranformations, known also as the Conjugate Duality Theorem, and showed its application in the economic context.

Therefore, using the heuristic approach in the economic context we have derived a result which is consistent with the Conjugate Duality Theorem. So far we have shown that it is valid in a one-dimensional case. In what follows we will show that it holds also for higher dimensions.

\section{Costs and profit}

The analysis in this chapter is based on the dual description of convex sets that can be represented as the intersection of supporting half-spaces. By comparing an active and a passive producer we derive the property of convexity of the maximum profit function as well as Hotelling's lemma. The graph of the minimum cost function is enveloped by its tangents and the minimum cost function is reconstructed graphically. It is important to emphasize that for this approach the nature and dimension of variables do not matter making the derived results completely general. These results include the convexity property of the Legendre-Fenchel transforms, the Conjugate Duality Theorem, and the properties of subdifferentiability and inversion.

Convex functions enable an elegant generalization of the notion of a marginal value ${ }^{3}$ and make the assumption of differentiability unneccessary. Submarginal value is defined as the slope of a tangent, the straight line that lies below the graph with which it has a common point. The possibility that there could be more such tangents allows for the vertical and horizontal parts of the curve connecting optimal values of the dual variables. In our example vertical parts describe the producer who at different prices supplies the

\footnotetext{
${ }^{2}$ Supremum as the lowest upper bound is a more general notion than maximum. It can include the maximum, the lowest finite upper bound that is not attained, or reach infinite values.

${ }^{3}$ It is generally understood that the expression "marginal value" refers to the first derivative of the function that is the object of analysis, e.g. cost function-marginal cost, production function-marginal product, and so on.
} 
same quantity. The producer who finds himself on the horizontal segment maximizes profit with different quantities of production. This does not change the essence of heuristic considerations. The assumptions on which we rely are convexity and closedness of the epigraph of the cost curve.

We will envelop the cost curve with tangents and show that the dual description of the epigraph of the cost curve shows the essence of the duality between minimum costs and maximum profit. Let us start with one of the prices that are determined by submarginal costs for a given quantity of production

$$
p^{0} \in \partial c\left(y^{0}\right)
$$

The corresponding tangent enables an interesting economic interpretation (Figure 4). Its equation is

$$
\begin{aligned}
& c-c\left(y^{0}\right)=p^{0}\left(y-y^{0}\right), \\
& c=p^{0} y-\left(p^{0} y^{0}-c\left(y^{0}\right)\right), \\
& p^{0} y^{0}-c\left(y^{0}\right)=p^{0} y-c .
\end{aligned}
$$

It connects all combinations of quantities of production and costs that give the same profit. This profit is represented by the absolute value of the segment on the c-axis ( 0 to $\left.-\pi\left(p^{0}\right)\right)$ in Figure 4.

That segment can not be greater because, if it could, the isoprofit line would not have common points with the cost curve. Consequently, we could not find an economically available combination of the quantity of production and costs. Therefore, maximum profit is given by,

$$
\pi\left(p^{0}\right)=p^{0} y^{0}-c\left(y^{0}\right)=\sup _{y} p^{0} y-c(y) .
$$


Figure 4: Legendre-Fenchel transformations of costs and profits

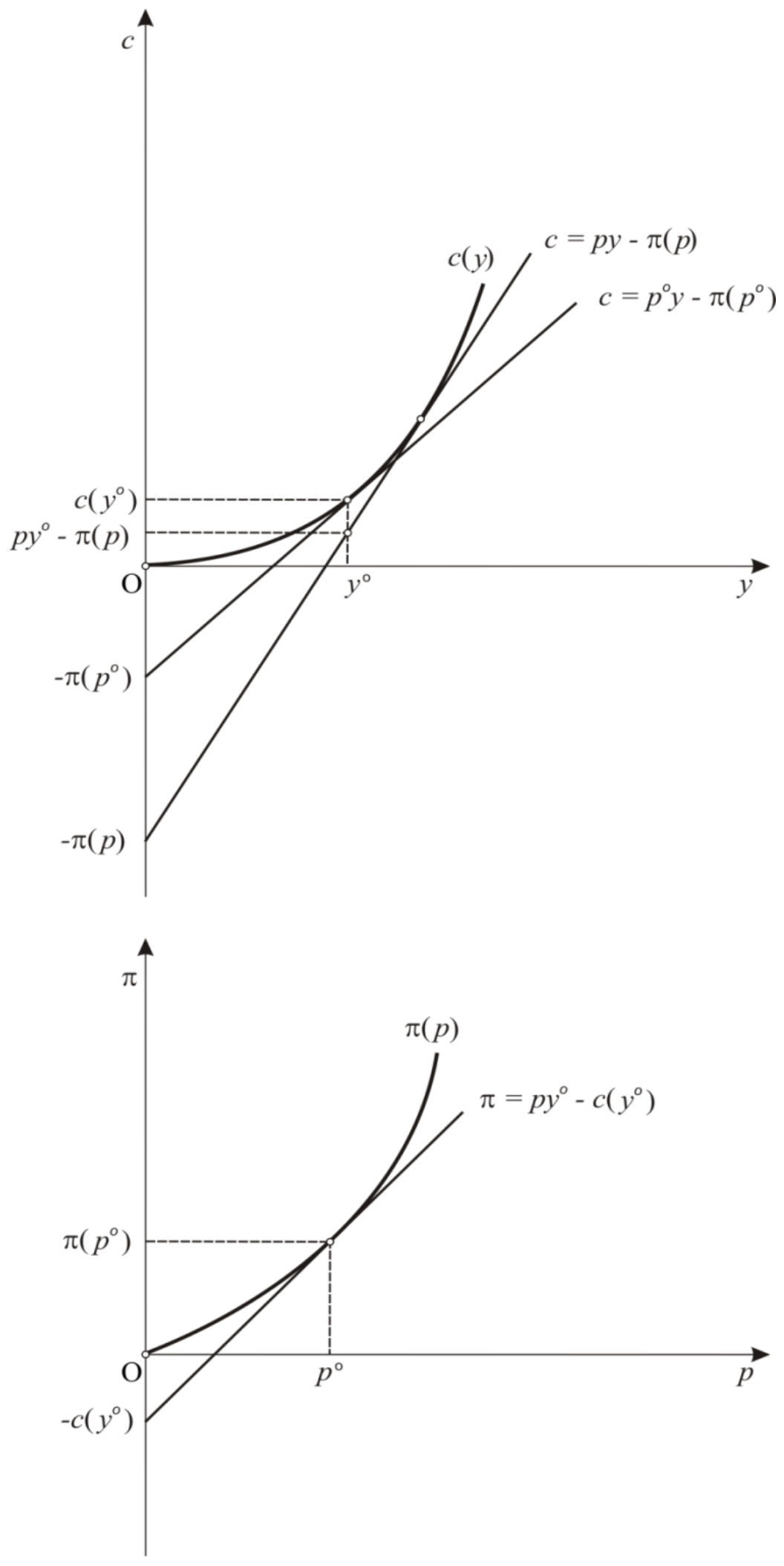

Source: Authors 
The solution to this optimization problem is the given level of production, $y^{0}$. If the price changes, the producer can act in one of two ways. He can either choose to produce the same quantity or to adjust to the market changes. An active producer follows the maximum profit curve. A tangent on the maximum profit curve leads the passive producer and his profit is given by

$$
\pi=p y^{0}-c\left(y^{0}\right)
$$

In dual space this tangent is, in fact, the isocost line. It contains all combinations of prices and profits for which costs are the same,

$$
\begin{aligned}
& \pi-\pi\left(p^{0}\right)=y^{0}\left(p-p^{0}\right), \\
& \pi=p y^{0}-\left(p^{0} y^{0}-\pi\left(p^{0}\right)\right),
\end{aligned}
$$

(10b)

$$
p^{0} y^{0}-\pi\left(p^{0}\right)=p y^{0}-\pi
$$

The relationship between the maximum profit curve and its tangent reveals the convexity of the maximum profit curve,

$$
\pi(p) \geq p y^{0}-c\left(y^{0}\right)
$$

The slope of the tangent or the original level of production represents the slope of the maximum profit curve and confirms Hotelling's lemma,

$$
y^{0} \in \partial \pi\left(p^{0}\right) .
$$

If a different price on the original level of production, $y^{0}$, is chosen, maximum profit becomes different from the real profit. The difference between them shows by how much costs would have to be lowered in order to maintain maximum profit, or

$$
\pi(p)-\left(p y^{0}-c\left(y^{0}\right)\right)
$$

$$
\pi(p)-p y^{0}+c\left(y^{0}\right)
$$

Costs would then be given by 


$$
p y^{0}-\pi(p) .
$$

Geometrically, this value is the coordinate of the point above the original level of production on the tangent to the cost curve determined by a different price. Economic efficiency does not permit further lowering of costs. Thus, minimum costs for the given level of production are equal to the maximum difference between total revenue and maximum profit,

$$
c\left(y^{0}\right)=p^{0} y^{0}-\pi\left(p^{0}\right)=\sup _{p} p y^{0}-\pi(p) .
$$

Profit is then also at its maximum level. The previous result follows from the dual description of the graph of the minimum cost function, whose graph is the upper envelope of its tangents. In this way we have reconstructed the cost function and derived the result which is consistent with the Conjugate Duality Theorem. The solution to the dual optimization problem (15) is the sub-marginal cost of the given level of production,

$$
p^{0} \in \partial c\left(y^{0}\right) .
$$

We should point out that we can obtain the solution to the dual problem as a submarginal value of the primal function, $c(y)$. Likewise, we can obtain the solution to the primal problem (8) as a sub-marginal value of the dual function, $\pi(p)$. This is the essence of the Legendre-Fenchel transformations and their sub-differentiability and inversion properties (expressions 12 and 16).

Particularly interesting is the situation where the cost function is not differentiable which is represented in Figure 5. Then we can draw more tangents through the kink on the cost curve. At prices that are sufficiently close to $p^{0}$ the optimum quantity is the same, $y^{0}$, and the maximum profit curve in that segment is linear. For that quantity of production the sub-marginal cost correspondence gives the interval of prices for which the sub-marginal profit is constant, meaning that the optimum production remains the same. 
Figure 5: The relationship between non-differentiability and linearity of the dual cost and profit functions

The same quantity of production optimal at different prices

Different quantities of production optimal at the same price
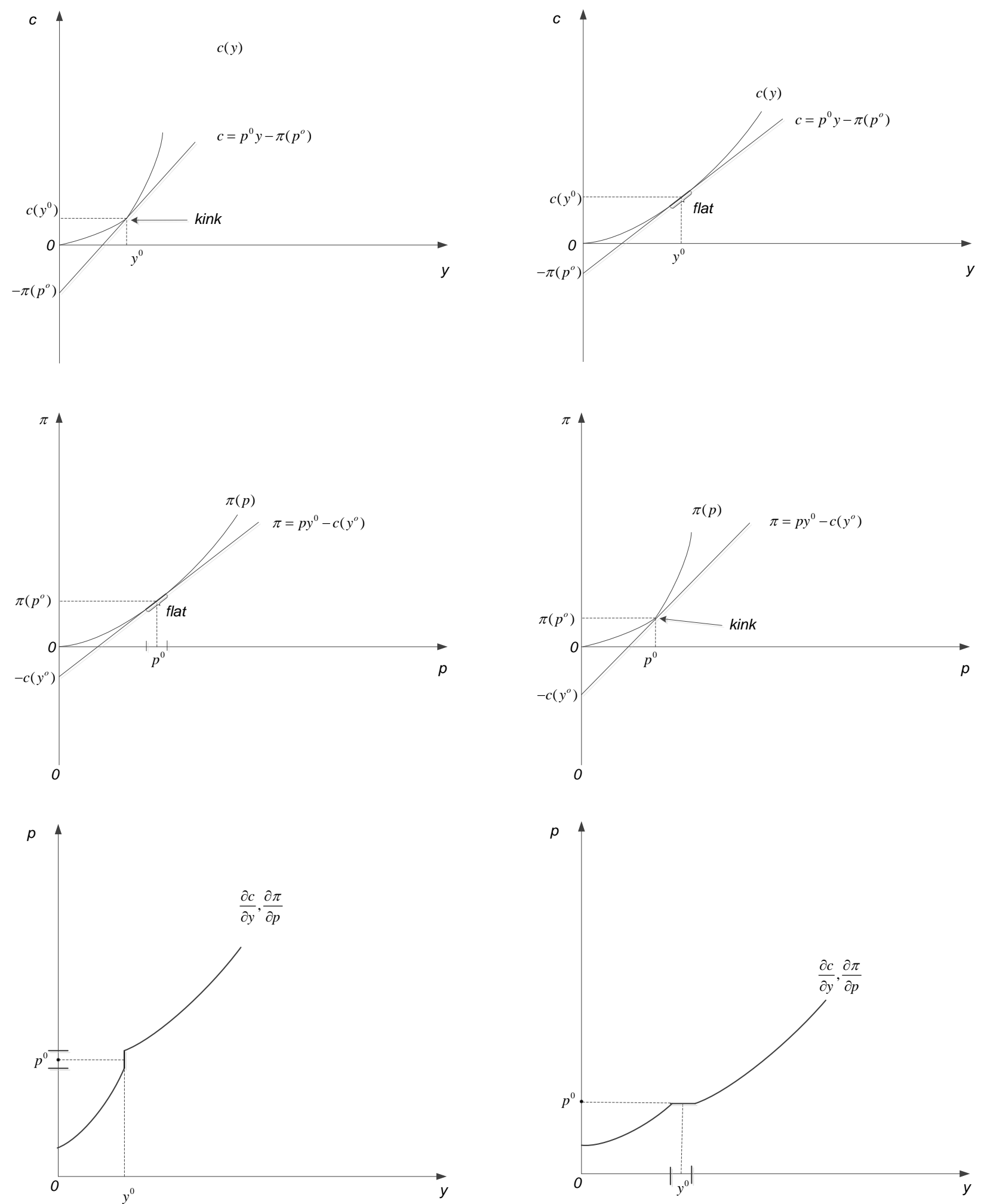

Source: Authors 
If the cost curve in the neighborhood of some production $y^{0}$ is linear, the tangent on the cost curve for sufficiently close quantities of production is the same. At the same price p0 different quantities of production are optimal and the maximum profit curve in this point has a kink. The sub-marginal profit correspondence gives the interval of the optimum quantities of production for which the sub-marginal costs are constant.

It is important to note that in this chapter neither the nature of the dual functions nor the dimensions of the dual variables are relevant. Manipulations with inequalities remain the same also in spaces of higher dimensions. The relations described by these inequalities do not change either. The only thing that happens is that curves become surfaces and tangents become tangential hyperplanes. The only assumptions that are used are convexity and closedness of the epigraph of the primal function.

\section{Legendre-Fenchel transformations}

The Hotelling's lemma or the sub-differentiability property indicate that the same curve connects optimum values of the dual variables. The perpendicular parts on that curve correspond to the spikes on the graph of the primal function and to the flat (linear) parts on the graph of the dual function. At the same time, horizontal parts correspond to the flat parts on the graph of the primal function and to spikes on the graph of the dual function. These are the geometrical consequences of the connections between sub-marginal values and solutions of the corresponding optimization problems.

The dimension of the dual variables was of no consequence either for the conclusions about convexity of the Legendre-Fenchel transformations, the Conjugate Duality Theorem, nor for the properties of inversion and differentiability. Spaces where dual variables "live" can be of any finite dimension and the results we obtained are valid also outside of the interior of the effective domain ${ }^{4}$. We will complement them with the properties of monotonicity and cyclical monotonicity of the subdifferential. They are the sets containing subgradients that are high-dimensional analogues of submarginal values.

It follows from (7a) that the equation of the tangent to the cost curve in $\left(y^{0}, c\left(y^{0}\right)\right)$ is

$$
c=c\left(y^{0}\right)+p^{0}\left(y-y^{0}\right) .
$$

The relationship between the cost curve and the respective tangent results in the following inequality:

$$
c\left(y^{1}\right) \geq c\left(y^{0}\right)+p^{0}\left(y^{1}-y^{0}\right) .
$$

\footnotetext{
${ }^{4}$ Effective domain is the set on which the function takes finite values.
} 
Similarly:

$$
c\left(y^{0}\right) \geq c\left(y^{1}\right)+p^{1}\left(y^{0}-y^{1}\right) .
$$

Adding those inequalities and multiplying by $(-1)$ we get the monotonicity of the subdifferential

$$
\left(p^{0}-p^{1}\right)\left(y^{0}-y^{1}\right) \geq 0 .
$$

Hence, $y^{i}$ is optimal for $p^{i}$. Consequently, higher prices will not result in lower quantities being offered on the market.

If we take into consideration more than just two tangents, by adding the respective inequalities we obtain

$$
p^{0}\left(y^{1}-y^{0}\right)+p^{1}\left(y^{2}-y^{1}\right)+\ldots+p^{n}\left(y^{0}-y^{n}\right) \leq 0 .
$$

In this inequality that expresses cyclical monotonicity, prices and quantities can trade places because the primal function in the analysis $c(y)$ can be replaced by the dual one $\pi(p)$ or,

$$
y^{0}\left(p^{1}-p^{0}\right)+y^{1}\left(p^{2}-p^{1}\right)+\ldots+y^{n}\left(p^{0}-p^{n}\right) \leq 0 .
$$

Instead of convex conjugates we can also observe concave Legendre-Fenchel transformations. In that case the sign in previous inequalities changes direction.

The properties that are derived have significant application not only in production theory but also in the theory of consumer behavior. These properties include the concavity of the expenditure function, the concavity of the cost function, as well as the convexity of the profit function. They are all consequences of the shape of the graph of the respective Legendre-Fenchel transformations. Shephard's lemma and Hotelling's lemma stem from the property of differentiability.

From the monotonicity of the subdifferential stem numerous comparative statics results. We should reinstate that the conditions of symmetry are tied to cyclical monotonicity while alternative ways of depicting tastes and technology are connected with the duality of Legendre-Fenchel transformations.

It has been abundantly clear that numerous applications of convex analysis in economics carry significant interpretations. Here we will direct our attention to the connection between profit and the set of production plans (the production set) to point out once more 
the essence of duality in applying the Conjugate Duality Theorem. The primal function is a convex indicator of a closed and convex set of production plans, $\mathrm{Y}$,

$$
f(\mathbf{y})=\left\{\begin{array}{cc}
0, & \mathbf{y} \in \mathbf{Y} \\
+\infty, & \text { otherwise }
\end{array} .\right.
$$

By applying the convex Legender-Fenchel transformation to $f(y)$ we obtain the profit function,

$$
\pi(\mathbf{p})=\sup _{\mathbf{y}} \mathbf{p y}-f(\mathbf{y})=\sup _{\mathbf{y} \in \mathbf{Y}} \mathbf{p y}
$$

Note that positive linear homogeneity of the profit function is also a common property of the Fenchel transformations of indicator-functions, expenditure function, and the cost function. The Fenchel transformation of the profit function brings us back to the indicatorfunction

$$
f(\mathbf{y})=\sup _{\mathbf{p}} \mathbf{p y}-\pi(\mathbf{p}) .
$$

In this problem the objective function is linearly homogeneous and the result has two values. If no price vector generates a positive value for the objective function, given its homogeneity, zero becomes its least upper bound. In the opposite case homogeneity leads us towards infinitely high values

$$
f(\mathbf{y})=\left\{\begin{array}{cc}
0, & \mathbf{p y}-\pi(\mathbf{p}) \leq 0 \\
+\infty, & \text { otherwise }
\end{array} \quad\right. \text { p }
$$

Therefore, the set of production plans has a dual description,

$$
\mathbf{Y}=\{\mathbf{y}: \pi(\mathbf{p}) \geq \mathbf{p y} \quad \forall \mathbf{p}\}
$$

Instead of describing the technologically feasible production plans directly, we did it indirectly by observing the intersection of all spaces that contain them. The dual description of the set of production plans follows from the Conjugate Duality Theorem.

We could summarize this by saying that applying the Conjugate Duality Theorem to indicator-functions is just the dual description of a set that is convex and closed. It is important to note that the dual description of the set of technologically feasible production plans shows that technology can also be described by the profit function. Clearly, the profit function is convex because it is a convex Legendre-Fenchel transformation of the respective indicator function, $f(\mathbf{y})$. 
To illustrate the power of the result in (26) let us look at the technology that uses labor, L, and capital, $\mathrm{K}$, to produce one product, $\mathrm{y}$. The primary description of the set of technologically feasible production plans is

$$
\mathbf{Y}=\{(-L,-K, y): L, K \geq 0, y \leq g(L, K)\},
$$

where $\mathrm{g}(\mathrm{L}, \mathrm{K})$ is a production function. Its dual description is

$$
\mathbf{Y}=\{(-L,-K, y): p y-w L-r K \leq \pi(w, r, p) \forall w, r, p\}
$$

where $w$ is wage, $r$ is the price of renting capital and $p$ is the product price. Because of the technological efficiency the production function describes maximum production for the given quantities of inputs,

$$
g(L, K)=\max _{y}\{y: p y-w L-r K \leq \pi(w, r, p) \forall w, r, p\} .
$$

For a technologically efficient production plan the previous inequality becomes an equality for prices that prevail in the production plan that satisfies economic efficiency. After normalizing the product prices from (29) it follows that

$$
g(L, K)=\min _{w, r} w L+r K+\pi(w, r, 1) .
$$

From the First Order Conditions we re-derive Hotelling's lemma,

$$
L=-\frac{\partial \pi}{\partial w}(w, r, 1), K=-\frac{\partial \pi}{\partial r}(w, r, 1)
$$

We should keep in mind that in reconstructing the production function we solve the previous system of equations using the prices of inputs.

This approach has another advantage. A proportional change in prices has a just proportional effect on the maximum profit. The tangential hyperplane to the graph of the indicator-function only rotates around the isoprofit line showing maximum real profit. This change that does not affect the profit maximizing quantities but only the maximum profit itself and it can be read off on the $\pi$ axis which is represented in Figure 6. 
Figure 6: Supporting hyperplane to the graph of the indicator-function and the homogeneity of the profit function

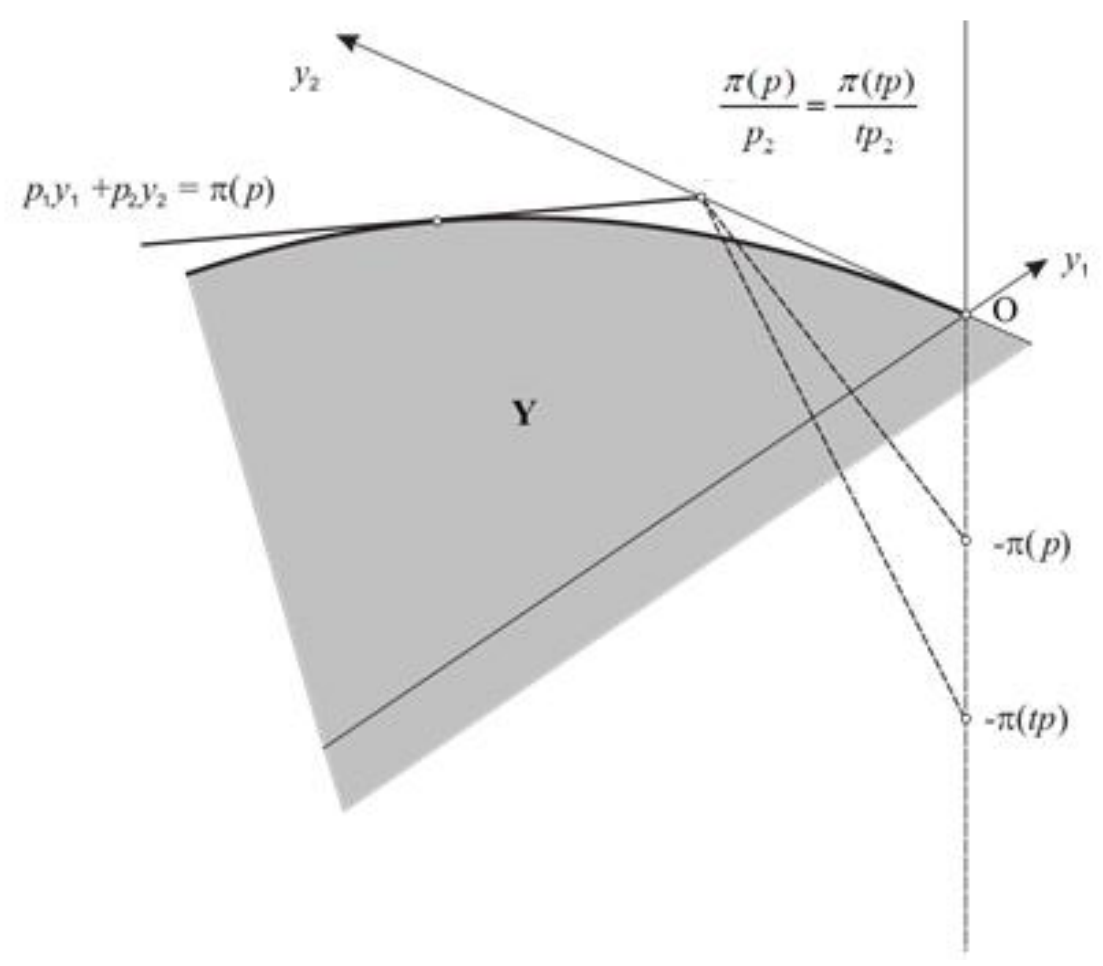

Source: Authors

Normal vector to the common hyper-plane to the graph of the profit function also shows the optimal quantities at proportional prices. It is clear that the product supply and input demand functions in perfectly competitive markets are homogeneous of the degree 0 . In fact, all we know about them and all we know about the profit function follows from the Legendre-Fenchel transformations. In addition, knowing that for the given product prices indirect utility function is the inverse of the expenditure function, the direct and indirect application of the properties of the Legendre-Fenchel transformations enables us to obtain all the results in the theories of demand as well as of production.

\section{Conclusion}

It is well-known that in perfect competition the optimum production is obtained by equating price and marginal cost. The aim of this paper was to show how, starting with that equality, it is possible to inductively derive general results of convex analysis from which, then, deductively follow the indirect and direct results of the theories of consumer and producer behavior. The point of departure was the standard economic problem of profit maximization for the firm in perfect competition. When the Fundamental Theorems 
of Calculus are applied to the marginal cost curve (which is also the supply curve) a new perspective is created. In this new perspective the marginal cost curve gains a new interpretation as the marginal profit curve. This is a novel way of deriving Hotelling's lemma. Based on heuristic considerations Hotelling's lemma is interpreted as the First Order Condition of the optimization problem from which the cost function is reconstructed. In this way, by using the unique interpretations of marginal profit and novel geometry in the economic context, the dual of the profit maximization model is derived and the Conjugate Duality Theorem is confirmed.

The novel approach and methods used in this paper are strengthened by the generality of the results that are obtained. The assumptions on which the analysis rests are only convexity and closedness of the cost or primal function. Based on the dual description of the epigraph of the cost function in the economic context follow the results regarding the properties of the Legendre-Fenchel transformations, namely their convexity, duality, inversion, differentiability, monotonicity, and cyclical monotonicity.

The unique way from the well-known economic phenomenon to the general results in convex analysis is supplemented by applying the Conjugate Duality Theorem on the indicator function of the set of feasible production plans. This reveals the essence of duality in the dual description of the set of technologically feasible production plans by showing that profit function is just another way of describing technology. Another important result is that this makes it possible to reconstruct the production function, the process in which Hotelling's lemma is obtained as the necessary First Order Condition. It is interesting to note that application of the Conjugate Duality Theorem on the profit function enables graphical representation of the linear homogeneity of the profit function.

Both microeconomic theory and convex analysis are rich and seemingly complex. The literature that deals with applications of the analysis of convex sets can be made more accessible to the reader when those results are derived heuristically in the economic context. It could be said that the inductive-deductive approach used in this paper that analyzes the properties of Legendre-Fenchel transformations and their applications establishes a two-way connection between convex analysis and microeconomic theory.

\section{References}

Appelbaum, E. (1978). Testing Neoclassical Production Theory. Journal of Econometrics. 7 (1), pp. 87102. https://doi.org/10.1016/0304-4076(78)90007-6

Blackorby, C., Primont, D., Russel, R.R. (1978). Duality, Separability and Functional Structure. New York: American Elsevier.

Blume, L. E. (2008a). Convex Programming. In Durlauf, S. N.; Blume, L. E., eds. The New Palgrave Dictionary of 
Economics, New York: Palgrave Macmillan. https://doi.org/10.1057/978-1-349-95121-5_591-2

Blume, L. E. (2008b). Duality. In Durlauf, S. N.; Blume, L. E., eds. The New Palgrave Dictionary of Economics.

New York: Palgrave Macmillan. https://doi.org/10.1057/978-1-349-95121-5_285-2

Burgess, D. F. (1975). Duality Theory and Pitfalls in the Specification of Technologies. Journal of

Econometrics. 3 (2), pp.105-121. https://doi.org/10.1016/0304-4076(75)90041-X

Christensen, L. R., Jorgenson, D. W., Lau, L. J. (1971). Conjugate Duality and the Transcedental

Logarithmic Production Function. Econometrica. 39, pp. 255-256.

Cornes, R. C. (1992). Duality and modern economics. Cambridge: Cambridge University Press. https://doi.org/10.1017/CBO9780511571725

Diewert, W. E. (1971). An Application of the Shepard Duality Theorem: A Generalized Leontief Production

Function. Journal of Political Economy. 79 (3), pp. 481-507. https://doi.org/10.1086/259764

Diewert, W. E. (1982). Duality Approaches to Microeconomic Theory. In: Intriligator, M.D.; Arrow, K.J., eds.

Handbook of Mathematical Economics, pp. 535-599. Amsterdam: North Holland. https://doi.org/10.1016/S1573-4382(82)02007-4

Diewert, W. E. (1974a). Applications of Duality Theory. In: Intriligator, M.D; Kendrick, J.W., eds. Frontiers of Quantitative Economic, pp. 106-166. Amsterdam: North Holland.

Fenchel, W. (1953). On Conjugate Convex Functions. Canadian Journal of Mathematics. 1, pp. 73-77. https://doi.org/10.4153/CJM-1949-007-x

Fenchel, W. (1953). Convex Cones, Sets, and Functions. Princeton: Department of Mathematics, Princeton University.

Hotelling, H. (1932). Edgeworth's Taxation Paradox and the Nature of Supply and Demand Functions.

Journal of Political Economy. 40 (5), pp. 577-616. https://doi.org/10.1086/254387

Jorgenson, D. W. and Lau, L. J. (1974a). The Duality of Technology and Economic Behavior. The Review

of Economic Studies. 41 (2), 126, pp. 181-200. https://doi.org/10.2307/2296712

Lau, L.J. (1974). Applications of Duality Theory: a comment. In Intriligator, M.D., Kandrick, D.A., Frontiers of Quantitative Economics. Vol. II, pp. 176-199. Amsterdam: North-Holland.

Lau, L.J. (1978). Applications of Profit Function. In Fuss, M., McFadden, D., Production Economics: A Dual Approach to Theory and Applications. Vol II, Part IV, pp. 133-216. Amsterdam: North-Holland.

Lusk, J. L, Featherstone, A. M, Marsh, T. L. and Abdulkadri, A. O. (2002). Empirical properties of duality 
theory. The Australian Journal of Agricultural and Resource Economics. 46 (1), pp. 45-68. https://doi.org/10.1111/1467-8489.00166

Mas-Colell, A.; Whinston, M.D. and Green, J. (1995). Microeconomic Theory. New York: Oxford University Press.

McFadden, D. (1966). Cost, Revenue and Profit Functions: A cursory review. Institute for Business and Economic Research Working Paper no. 86, Berkeley, CA: University of California.

McFadden, D. (1978). Cost, Revenue and Profit Functions. In Fuss, M.; McFadden, D., eds.: Production Economics: A Dual Approach to Theory and Applications. North-Holland, Amsterdam.

Rockafellar, R.T. (1970). Convex Analysis. Princeton: Princeton University Press. https://doi.org/10.1515/9781400873173

Rosas, F. and Lence, S.H . (2015). Duality theory econometrics : How reliable is it with real-world data?.In

AAEA \& WAEA Joint Annual Meeting, San Francisco, California. Agricultural and Applied Economics Association \& Western Agricultural Economics Association.

Roy, R. (1942). De l' utilite. Paris: Hermann.

Sargan, J. D. (1971). Production Functions. In Layard, P. R. G., Sargan, J. D., Ager, M. E., Jones, D. J. Qualified Manpower and Economic Performance. London: Penguin Press.

Shepard, R.W. (1970). Theory of Cost and Production Functions. Princeton: Princeton University Press.

Shumway, C. R. (1995). Recent Duality Contributions in Production Economics. Journal of Agricultural and Resource Economics. 20 (1), pp.178-194.

Uzawa, H. (1964). Duality Principles in the Theory of Cost and Production. International Economic Review.

5, pp. 216-220. https://doi.org/10.2307/2525564

Weymark, J. A. (1980). Duality Results in Demand Theory. European Economic Review. 14, pp. 377-395. https://doi.org/10.1016/S0014-2921(80)80008-0 